\title{
DESIGN AND IMPLEMENTATION OF ACULOPS LYCOPERSICI POPULATION DYNAMIC MODEL PROTOTYPE BASED ON CELLULAR AUTOMATA
}

\author{
Shuai Zhang *, Dongsheng Wang, Linyi Li, Yongda Yuan \\ Center of Information Technology in Agriculture, Shanghai Academy of Agriculture Sciences, \\ Shanghai, P. R. China 201106 \\ * Corresponding author, Address: Center of Information Technology in Agriculture, Shanghai \\ Academy of Agriculture, Shanghai 201106, P. R. China, Tel: +86-21-62200281, Fax: \\ +86-21-62204989, Email: zhangshuai@lreis.ac.cn
}

Abstract: Faced upon the research status of Aculops lycopersici, the importance of population dynamic has been put forward. The feasibility and superiority of cellular automata applied in the simulation of Aculops lycopersici has been discussed. This paper has put forward an Aculops lycopersici population dynamic model prototype based on cellular automata, the result showed that this model can be used to simulate the dynamic population of Aculops lycopersici. When it is applied, the improvement of parameter should be considered, at the same time, this model could provide reference for the simulation of other species of insect.

Key words: Aculops lycopersici, cellular automata, population dynamic

\section{INTRODUCTION}

Aculops lycopersici is classified as Arthropoda, Arachnida (Acari: Eriophyidae). It has been first discovered in Australia (Tryon, 1937), and until 1986 there ware 47 countries which has reported the occurrence of Aculops lycopersici (Nemoto, 2000). Now Aculops lycopersici has became a world wide serious pest with tomato as main host (Jeppson et al., 1975).

Please use the following format when citing this chapter:

Zhang, S., Wang, D., Li, L. and Yuan, Y., 2009, in IFIP International Federation for Information Processing, Volume 294, Computer and Computing Technologies in Agriculture II, Volume 2, eds. D. Li, Z. Chunjiang, (Boston: Springer), pp. 1319-1328. 
Aculops lycopersici has been first reported in China by Kuang of Nanjing Agriculture University in 1983(Kuang et al., 1983). In 1999 Chen made an introduction of harm on the protected tomato by Aculops lycopersici in Kunming (Chen, 2000). As the harm to the protected vegetable by Aculops lycopersici has became more and more serious, some scholars has do researches on the physiological characteristics, ecological characteristics and control techniques of Aculops lycopersici. The impact of temperature and relative humidity on the growth and propagation of Aculops lycopersici has been researched (Xu et al., 2006). Wu has done the research of the impact to the tomato plants by Aculops lycopersici (Wu et al., 2006). Zhang has made clear the distribution of Aculops lycopersici in China (Zhang et al., 2007). And the work of biological control and chemical control has been well developed (Xu, 2006).

Many works have been done about Aculops lycopersici, but the rule of population dynamic of Aculops lycopersici is still unknown to us. Without the knowledge of population dynamic, the control work of Aculops lycopersici would stay the passive prevention stage. As there are many works about Aculops lycopersici which provide a good basis to research of population dynamic, if the research of population dynamic about Aculops lycopersici has been done, the result will be quite important to Aculops lycopersici, and the control work of Aculops lycopersici would go up to initiative prevention stage.

Now there are many scholars has do the population dynamic research about other kinds of insect pests to predict the occurrence of them. The methods which have been used are as follows:

1) Mathematics model

Logistic model: Some scholars predicted the number and area of insect pests by use logistic equation (Shen et al., 1985; Cui et al., 1998), the relationship between insect pests and environment factors could be generalization by analysis of insect pests occurrence system data, and the insect appearance area, damaged extend, population density can be predicted (Paul et al., 2004; Baumgartner, et al., 1998).

Matrix model: Matrix model can used to simulate the respond to environment of insect pests in different life stages (Leslie, 1945). Based on the Leslie matrix model, the variable dimension has been developed (Pang et al., 1980; Xu et al., 1981).

Mathematical equation: Differential equation and difference equation are availability tools to simulate the population dynamic of insect pests. Zhang has derived the nonlinear partial differential equation to describe the population dynamic of insect based on the principles of density dependence (zhang et al., 2001). Ruesink has simulated the population dynamic of alfalfa weevil by use difference equation, after that other scholars has improved this model to make the result more realistic (Wu et al., 1990; Zhang et al., 1994). 
This method has higher accuracy because of advanced mathematic method, occurrence period, occurrence area and damage degree can be predicted by use this method, but it only can predict the change of quantity, the spatial difference couldn't be expressed.

2) GIS Technology

Spatial analysis of GIS (Geographic Information System): It is availability method to research the spatial distribution of insect pests by analysis the relationship between geographic condition, climate resources and occurrence of insect pests (Shepherd et al., 1988; Schell et al., 1997).

Geo-statistics Combined with GIS: Some scholars also use GIS combined with geo-statistics to simulate the occurrence area of insect pests. The spatial missing data could be supplemented, and the result is more exact.

This method makes the distribution of insect pests simple and clear, but it is suit for researches in large scale, the relationship between environment factors and population dynamic of insect pests is not quantitative, though geo-statistics can make the damage degree quantitative, the result is at current time, the state in future couldn't be predicted.

3) Complex system modeling

Agriculture system is a complex system. The population dynamic of agricultural insect pests is related with many factors. As the development of information technology, the application of complex system modeling has became more and more extensive.

Artificial Neural Networks: Artificial Neural Networks has a good effect in predicting the trend of insect occurrence (Chon et al., 2000). Drake has applied BP Neural Network in the prediction of Plague Locust in Australia (Drake, 2001). Wei has put forward a predict method of agricultural insect pests base on fuzzy neural network, the result showed that this method is easy to use, the predict result is accuracy and it has extensive value in use (Wei et al., 2007).

Intelligent agent: Agent is a focus of artificial intelligence. It has been applied in the simulation of insect pests. Parry established a model based on model to simulate the number of aphid in cropland, each aphid was considered as an agent, the propagation, growth, death, and movement of aphid was simulated. Some domestic scholars also use agent to establish the population dynamic model of agricultural insect (Sa et al., 2005).

Cellular automata: Cellular automata have extensive applications in other field, such as the simulation of traffic flow (Zhou et al., 2005) and the change of land use (Fang et al., 2005), and it also has been used in simulation of plant invasion and forest insect pests. Cole $\mathrm{V}$ has established a model by using GIS and cellular automata to simulate the invasion of plant, it is a random parameters model and the species is not definite, so this model has a universal adaptability (Cole et al., 1999). Zhou G has simulate the break out of gypsy moth by cellular automata, four different ways was used 
to implement the cellular automata model, and the result shows that the effect of inverse distance weighted model is more accurate (Zhou et al., 1995).

The mathematic model can express the quantitative change quite well, but the spatial change of insect pests couldn't be expressed by it. While this problem can be solved by use GIS technology, GIS is good at space expression, but the quantitative change couldn't be described well, combined with geo-statistic the quantitative change could be described while the change is spatial, temporal change is hard to be expressed by this method. Complex system modeling can simulate the complex system accurately, and it is easy to use. But the Artificial Neural Networks is also couldn't express spatial change, and Intelligent agent put more attention on the behavior of the agent, the relationships between agent and experiment, agent and agent are not expressed well.

Cellular automata is a kind of complex system model It can express the spatial change and temporal change quite well. There is two state of a cell: live and die. The state of cell would be influenced by neighbor cell and the environment. The mode is simile to the occurrence of insect pests, especially Aculops lycopersici is a kind of micro insects which couldn't be observed by eyes, it is a problem to use other method, but it can be solved by cellular automata (the concrete method is introduced in next paragraph). So, cellular automata can be used to simulate the population dynamic of Aculops lycopersici.

\section{DESIGN OF ACULOPS LYCOPERSICI POPULATION DYNAMIC MODEL PROTOTYPE}

The model prototype is made up of three main parts: tomato plants, Aculops lycopersici and environment factors. Tomato plants can be ingested by Aculops lycopersici, and environment factors impact the growth of tomato plants and Aculops lycopersici.

This model described the dynamic change of Aculops lycopersici in a 2dimension space.

Aculops lycopersici is a kind of micro insect pests, so the body of Aculops lycopersici couldn't be considered as the basic cell, the grid with the size of 10 centimeters * 10 centimeters was considered as the basic cell in the model. In the range of a cell, only the growth state and number of Aculops lycopersici were considered, the position of Aculops lycopersici was not be considered, so the spatial change of Aculops lycopersici only could happened between each cells. Day is the basic time unit of the model. 


\section{$2.1 \quad$ Object}

\subsubsection{Tomato plants}

Nutrition is abstracted from tomato plants, and the change of nutrition will represent the growth process of Aculops lycopersici.

\subsubsection{Aculops lycopersici}

The duration of Aculops lycopersici has contain four states: egg, larva, nymph and adult.

Egg has a certain survival rate, survival eggs will grow to larva after egg stage, the

Larva has a certain survival rate, survival larva will grow to nymph after larva state, and larva can absorb the nutrition.

Nymph has a certain survival state, survival nymph will grow to adult after nymph stage, and nymph can absorb the nutrition of tomato plants. Nymph also has the ability of diffusion which egg and larva don't have.

Adult has a certain survival rate, and the survival adult has ability of spawning. And the same to the nymph, adult can absorb the nutrition, and has the ability of diffusion.

\subsubsection{Environment factors}

The environment factors of this model are mainly the temperature and relative humidity, which has an important impact on the process of tomato plants growth and Aculops lycopersici growth, diffusion.

\section{$2.2 \quad$ Rules}

According to the growth, diffusion process of Aculops lycopersici and the growth process of tomato plants, the rules of cellular automata have been established.

The rules of the model prototype based on cellular automata are composed by development rule, propagation rule, extinction rule, diffusion rule, tomato growth rule. The concrete contents of these rules would be different to Aculops lycopersici in different stage.

Development rule: The developmental threshold temperature (DTT) of the egg is $10.51^{\circ} \mathrm{C}$, the relationship between egg stage and temperature is $\mathrm{Y}$ (egg stage $)=0.0228 \mathrm{~T}$ (temperature) -0.2343 , the relationship between egg stage and relative humidity is $\mathrm{Y}$ (egg stage) $=0.4387-0.1397 \mathrm{RH}$ (relative 
humidity); The DTT of the larva is $9.02^{\circ} \mathrm{C}$, the relationship between larva stage and temperature is $\mathrm{Y}$ (larva stage) $=0.0148 \mathrm{~T}$ (temperature) -0.0935 , the relationship between larva stage and relative humidity is Y (egg stage) $=0.4449-0.1310 \mathrm{RH}$ (relative humidity); The DTT of the nymph is $9.02^{\circ} \mathrm{C}$, the relationship between nymph stage and temperature is $\mathrm{Y}$ (nymph stage) $=0.0148 \mathrm{~T}$ (temperature) -0.0935 , the relationship between nymph stage and relative humidity is $\mathrm{Y}$ (nymph stage) $=0.4449-0.1310 \mathrm{RH}$ (relative humidity).

Propagation rule: Only adult has the ability of oviposition. The relationship between oviposition period and temperature is $\mathrm{Y}$ (oviposition period) $=-1.0274 \mathrm{~T}$ (temperature) +47.508 , the relationship between oviposition period and relative humidity is $\mathrm{Y}$ (oviposition period) $=0.098$ $\mathrm{RH}$ (relative humidity) +12.536 ; the relationship between fecundity and temperature is $\mathrm{Y}$ (fecundity) $=2.3367 \mathrm{~T}$ (temperature) $-17.313(\mathrm{X}<26), \mathrm{Y}$ (fecundity) $=-3.007 \mathrm{~T}$ (temperature) $-122.9(\mathrm{X}>26)$.

Extinction rule: Egg has a certain survival rate, only survival eggs will grow to larva after egg stage, and the survival rate of egg is $95 \%$, the survival rate of larva is $85 \%$, the survival rate of nymph is $85 \%$, the survival rate of adult is $80 \%$. The adult will die after oviposition.

Diffusion rule: if the total number of Aculops lycopersici in a cell has reached the threshold, the nymph and adult will diffuse to the cells in which there is enough nutrition around the center cell, and when the nutrition in a cell in not enough for the Aculops lycopersici in it, the egg and larva will die, and the nymph and adult will diffuse to the cells in which there is enough nutrition around the center cell.

Tomato growth rule: set the initial nutrition of tomato plants is 100 thousand unit of nutrition. And the relationship between tomato nutrition and temperature is $\mathrm{Y}=50000+(\mathrm{X}-20) * 2500$. The nutrition growth would stop when it reached

Nutrition ingested rule: each larva ingests 3 units of nutrition a day; each nymph ingests 4 units of nutrition a day; each adult ingests 5 units of nutrition a day.

\subsection{Steps}

Step 1: the growth of tomato. According to the tomato growth rule and the value of tomato nutrition in each cell at last time, the new value of the tomato nutrition in each cell has been determined, and the initial distribution map of tomato nutrition has been formed.

Step 2: the growth of Aculops lycopersici. According to the development rule and propagation rule and the number of Aculops lycopersici in each cell at last time, the number of Aculops lycopersici at different growth periods in 
each cell has been determined, and the initial distribution map of Aculops lycopersici has been formed.

Step 3: the diffusion of Aculops lycopersici. According to the nutrition ingested rule and the initial distribution map of Aculops lycopersici, the quantity of nutrition that would be ingested by Aculops lycopersici can be determined, and it will be overlapped with the initial distribution map of tomato nutrition, the final distribution map of tomato nutrition has been formed after that, and the final distribution map of Aculops lycopersici also can be formed according to the diffusion rule.

\section{IMPLEMENTATION OF ACULOPS LYCOPERSICI POPULATION DYNAMIC MODEL PROTOTYPE}

The model prototype is implemented using ArcGIS Engine 9.1, the integrated development environment is Visio Studio.Net 2005.

Set the space is 25 meters * 30 meters, and the tomato plant in the center is set as the initial position of Aculops lycopersici. And the number of Aculops lycopersici is 40000 , the temperature is $26^{\circ} \mathrm{C}$, the relative humidity is $53 \%$.

According to the rules and steps above, the state of the 40000 Aculops lycopersici in 60 days has be simulated, the result of the model is showed in Fig. 1 and Fig. 2 as follows.

In the 60 days, there are three peaks of Aculops lycopersici population. About 55 days later, the number of Aculops lycopersici has reached maximum. Correspond in Fig.2, the distribution of Aculops lycopersici has get a maximum area in the 50th days, after that many tomato plants died because of Aculops lycopersici, and has became more centralized as showed in the 60th day.

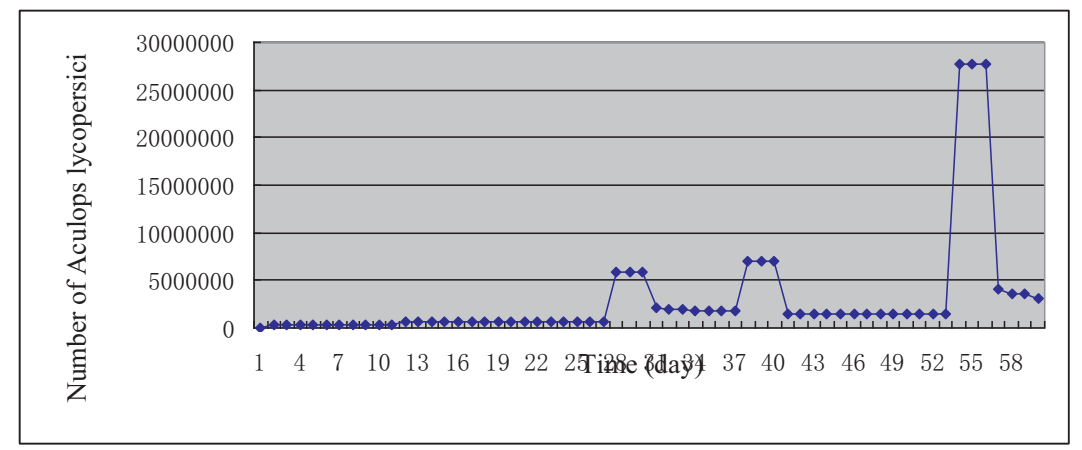

Fig.1. The quantitative change of Aculops lycopersici 

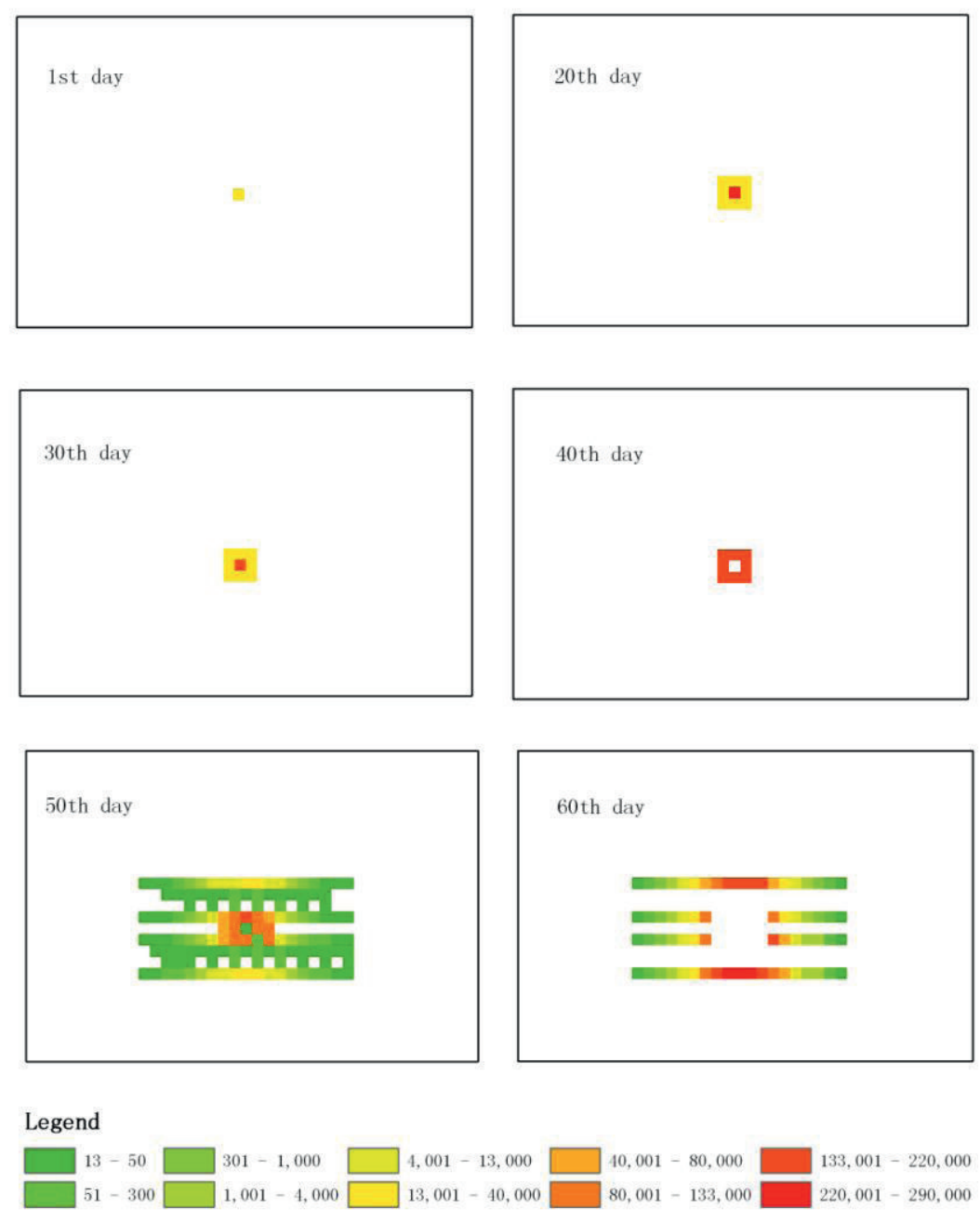

Fig.2. The distribution change of Aculops lycopersici

\section{CONCLUSION}

The result of this research has revealed that cellular automata can be used to simulate the dynamic population of Aculops lycopersici. Both the spatial change and temporal change could be described well in the model, and the data needed in the model is easy to obtain. A future research is the parameterization of the model and the validation of the model. 


\section{ACKNOWLEDGEMENTS}

We are grateful to colleagues who did the experiment of Aculops lycopersici and provide the data of ecological characteristics about Aculops lycopersici - the plant protection research group in Eco - Environment and Plant Protection Institute, Shanghai Academy of Agricultural Sciences. This work is supported by Extension and Application of vegetable insect and diseases diagnoses, prevention and cure expert system Project (Contract Number: 075119N85) of National Transformation of Agricultural Science and Technology Achievement Fund Program.

\section{REFERENCES}

Baumgartner, et al. Quantitative analysis of gypsy moth spread in the central appalachians. Population and Community Ecology for Insert Management and Conservation, 1998:99110.

Chen Bin, Luo Youzhen, Yin Suigong. A new insect pests of tomato - Aculops lycopersici. Yunnan Agricultural Science and Technology, 2000, 2:32-33. (in Chinese)

Chon TaeSoo, Kim JaMyung, Kim JaMyung. Use of an artificial neural network to predict population dynamics of the forest pest pine needle gall midge (Diptera:Cecidomyiida). Environmental Entomology. 2000, 29(6): 1208-1215.

Cole V, et al.. Modelling the spread of invasive species paramter estimation suing cellular automata in GIS, 1999. Department of Geography University of Auckland, New Zealand.

Drake. Use of remote sensing and ANN in prediction of pets in Queensland. Remote Sensing of Environment, 2001, 12(4):32-35.

Fang S.F., Gertnera G.Z., et al.. The impact of interactions in spatial simulation of the dynamics of urban sprawl, 2005. Landscape and Urban Planning.73: 294-306.

Jingan Cui, Lansun Chen. The effect of diffusion on the time varying logistic population growth. Computers \& Mathematics with Applications, 1998, 36(3):1-9.

Kuang haiyuan. Two new species and a new record species of gall mites from China. Acta Zootaxonomic Sinica, 1983, 8(4): 389-391. (in Chinese)

L.R.H. Jeppson, H.H. Keifer, E.B. Baker. Mites Injurious to Economic Plants, University of California Press, Berkeley, 1975, 614.

Leslie P H. On the use of matrices in certain population mathematics. Biometrika, 1945, 33: 183-211.

Pang Xiongfei. On The Use of Population Matrix Models For The Studies of Insect Ecology. Journal of South China Agricultural University, 1980, 1 (3) : 27-37. (in Chinese)

Paul W F, et al. Simulation model of Rhyzopertha dominica population dynamics in concrete grain bins. Journal of stored products research, 2004, 40:39-45.

Sa Li, Xiong Fanlun, Ding Jing, Zuo Honghao. An Agent-based Cellular Automata Models for Agroecosystems, Jouranl of University of Science and Technology of China, 2005,35(2):270-276.

Schell S P, J A Lockwood. Spatial analysis of ecological factors related to rangeland grasshopper (Orthoptera: Acrididae) outbreaks in Wyoming. Enbiron Entomol, 1997, 26(6):1343-1353. 
Shen Zuorui. The Modification of Logistic Fomula and Its application in Vegetable Aphides. Journal of Beijing Agricultral University, 1985, 11(3): 297-304. (in Chinese)

Shepherd R F. Proc Lymantriidae. A comparison of features of new and old world tussock moths. Washington DC: USDA, 1988.

Wei Mingshe, Guo Yong. Prediction of Crops Pests Level Based on Fuzzy Neural Network. Journal of Taiyuan University of Science and Technology, 2007, 28 (6) : 442-445. (in Chinese)

Wu Juan, Li Linyi, Xu Xiang, Yang Yizhong, Wang Dongsheng. Physiological Variation of Damaged Leaves of Tomato by Aculops lycopersici. Acta Horticulturae Sinica, 2006, 33(6): 1215-1218. (in Chinese)

Wu Zhongfu. Research on Simulating The Dynamic of Nilaparvata lugens Population. Journal of Fujian College of Agriculture, 1990, 19(2):115-122. (in Chinese)

$\mathrm{Xu}$ Rumei. Application of A Dimension-Changeable Matrix Model on The Simuluation of The Population Dynamics of Greenhouse Whiteflies. Acta Ecologica Sinica, 1981, 1 (2) : 147-158. (in Chinese)

Xu Xiang, Li Linyi, Wang Dongsheng, Hong Xiaoyue, Wu Juan, Yuan Yongda, Xie Xianchuan. Effect of temperature and relative humidity on development and reproduction of the tomato russet mite, Aculops lycopersici (Massee)(Acarina, Eriophyidae). Acta Entomologica Sinica, 2006, 49(5): 816-821. (in Chinese)

$\mathrm{Xu}$ Xiang. Ecology and Control of the Tomato Russet Mite Aculops lycopersici(Tryon). Nanjing Agricultural University, Master Degree Thesis. (in Chinese)

Zhang Shuai, Li Linyi, Wang Dongsheng, Zhao Jingyin. Research on Adaptive Distribution of Aculops Lycopersici in China, Proceedings of the 4th International Symposium on Intelligent Information Technology in Agriculture(ISIITA), 2007,491-494.

Zhang Wenjun, Gu Dexiang. A Non-Linear Partial Differential Equation to Describe Spatial and Temporal Changes of Insect Population. Ecological Science, 2001, 20(4): 1-7. (in Chinese)

Zhang wenqing, Gu Dexiang, Pu Zhelong. Improvement In The Method For Simulating The Dynamic of Insect Population: A Study on The Dynamic Population Simulation Model of Paddy Stem Borer (Tryporyza Incertulas Walker). Acta Ecologica Sinica, 1994,14(3):281289. (in Chinese)

Zhou G, et al, Forecasting the spatial dynamics of gypsy moth outbreaks using cellular transition models. Landscape Ecology, 1995, 10 (3): 177-189.

Zhou Zili, Wang Xinwei, Wang Yanna. Simulation System of City Traffic Flow Based on Cellular Automata. Computer Engineering, 2005, 31(13): 183-185. (in Chinese) 